\section{Die Laden der}

\section{Chirurgischen Societät der Stadt Bern}

R. Streit

Im Jahre 2000 erwarb die Ärztegesellschaft des Kantons Bern in einem Zürcher Antiquitätengeschäft die Lade der Chirurgischen Societät der Stadt Bern, eine kleine Truhe, die die Wappen und namentlichen Bezeichnungen von drei Obmännern mit den Jahrzahlen 1717, 1748 und 1766 trägt. Ein Bär mit der Stange, auf der die phrygische Mütze prangt, hält einen Wappenschild, auf dem Hand und Auge über einer geflügelten Kugel dargestellt sind, entsprechend dem Wahrspruch der Scherermeisterschaft: "Hic manus et oculus», von mir frei übersetzt: "Hier kommt es auf Hand und Auge an".

Die Angaben über die drei Obmänner lassen vermuten, dass die Lade 1717 angefertigt wurde und bis um 1766 in Gebrauch war. Samuel Gruber, AltOberspitalmeister, Burger und Mitglied des grossen Rats der Stadt Bern, "dismahliger Herr Obmann 1717», stand der Societät von 1698 bis 1720 vor. Albrecht Kastenhofer, "dismahliger Herr Obmann 1748", amtierte von 1748 bis 1757, der Inselchirurg Herr Johann Jakob Hartmann, "der Zeit Obmann 1766», von 1763 bis 1775 .

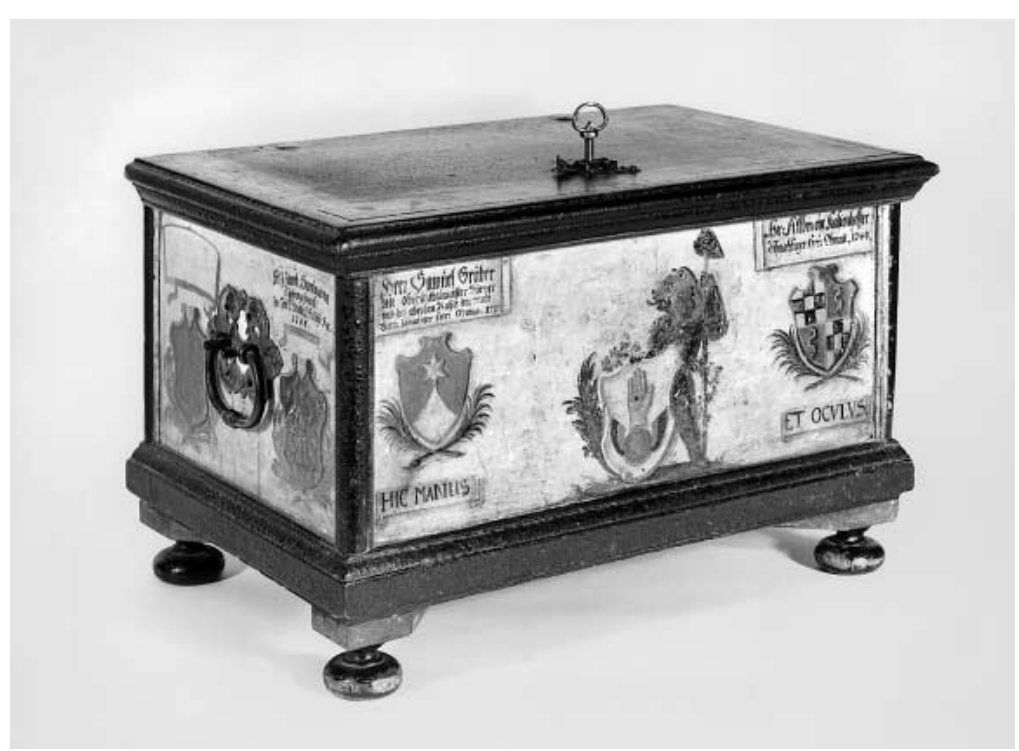

Berner Chirurgenlade. Burgerbibliothek Bern.

Korrespondenz:

Dr. med. Reinhold Streit

Thunstrasse 62

CH-3400 Burgdorf
Bei unserer Lade handelt es sich höchstwahrscheinlich um die Vorgängerin der Lade, die 1764 auf Bestellung der Societät vom Ebenisten Funk aus der berühmten Kunstschreinerfamilie angefertigt worden ist und sich jetzt als Leihgabe der Ärztegesellschaft im Bernischen Historischen Museum befindet.

Die Lade versinnbildlicht die Selbständigkeit der Meisterschaft und stellt ein Symbol ethischer Werte dar. Sie diente zur Aufbewahrung der Gesellschaftsakten und des Vereinsvermögens. Beispielsweise musste jedes Mitglied für jeden Lehrling einen Betrag einzahlen. Die Gebühren für die Meisterprüfung waren hoch. Auch durfte, mindestens zu gewissen Zeiten, die Meisterschaft Bussen bei den Empirici und Stümplern einziehen. Dabei handelte es sich um Kurpfuscher ohne Ausbildung und ohne bestandene Prüfung.

Zum Gesellschaftsgut gehörten die Freibriefe für die Meisterschaft der Scherer und Wundärzte der Stadt Bern. Die erste Fryung der Meisterschaft Schererhandwerks datiert vom Jahre 1502. Später sind weitere Ordnungen des Handwerks mit entsprechenden Eidesformeln festgelegt worden. In der Lade aufbewahrt wurden das 1765 von der Societät angeschaffte neues grosse Siegel, ferner goldene Becher, die bei besonderen Anlässen, z.B. nach erfolgter Rechnungsablage, die Runde machten und zu Ehren der ganzen Societät und auf die Gesundheit des Herrn Obmanns geleert wurden. 50 Taler aus der Gesellschaftslade plus Sponsorbeiträge ermöglichten es 1775, aus den alten Bechern einen neuen Goldpokal in getriebener Arbeit anfertigen zu lassen, der mit dem Siegel der Societät und den Wappen der damaligen Mitglieder und des Sekretärs verziert war.

Die Chirurgische Societät der Stadt Bern vereinigte als Meisterschaft die handwerklich ausgebildeten Wundärzte; die gelehrten Doktoren der Medizin gehörten ihr nicht an. Sie hatte ausschliesslich berufliche Belange zu regeln. Sie befasste sich mit der Ausbildung der Lehrlinge, der Zulassung zur Meisterprüfung, also der Verleihung des Meistertitels, aber auch mit Tariffragen. Um Politisches hatte sie sich im patrizisch regierten Bern nicht zu kümmern. In Zürich dagegen gehörten die Scherer zusammen mit den Badern zur Gesellschaft zum Schwarzen Garten, die ihrerseits Teil der Schmiedenzunft war und damit im Grossen Rat vertreten war.

$\mathrm{Zu}$ den Zürcher Kollegen bestand stets ein guter Kontakt. In allen Städten, so auch in Bern, kam es immer wieder $\mathrm{zu}$ Auseinandersetzungen mit den Badern, die in ihren Badstuben nicht nur zum Teil rasierten, sondern auch zur Ader liessen. Das war ihnen aber nur unter bestimmten Bedingungen und mit einer definierten Methode erlaubt. Auf Anfrage der Berner Societät bei den Zürcher Kollegen, ob die Bader dort zu Ader lassen dürften, weil sie ja in der gleichen Gesellschaft seien, reagierten diese scharf. Sie erklärten, sie hätten selber einen eigenen Obmann und eine eigene Lade, seien also selbständig.

1707 wurde eine Landmeisterschaft in der Region Langenthal, gewissermassen eine Vorläuferin des heutigen Bezirkvereins Oberaargau, für die Ämter 
Wangen, Aarwangen, Bipp und Huttwil gebildet. Die Meister dieser Gegend wünschten eine Lade unter sich aufzurichten und erbaten dazu die Bewilligung der Bernischen Meisterschaft. Bern stimmte zu mit zahlreichen Auflagen. Dabei wurde festgehalten, dass, falls die Mitgliederzahl der Langenthaler Societät unter vier sinken würde, die Gesellschaft aufzuheben sei. Die Lade müsse in diesem Falle nach Bern abgeliefert werden.

Bei Diskussionen mit Gesellen oder mit Meistern und bei Massregelungen derselben, wo heftige Auseinandersetzungen zu erwarten waren, fanden solche Verhandlungen "vor offener Lade" statt. Das verlieh den Verhandlungen eine gewisse Feierlichkeit. Mass und Anstand sollten gewahrt bleiben.
So hatte die Lade die Bedeutung eines Zeichens von Unabhängigkeit und Selbständigkeit. Sie versinnbildlichte aber auch Standesbewusstsein, ethische Grundsätze, Ehrbarkeit und Disziplin.

\section{Literatur}

- Schneebeli M. Handwerkliche Wundarzneikunst im alten Bern. Bern; 1949. - Das alte Archiv, dem Schneebeli seine Angaben entnommen hat, wird in der Burgerbibliothek Bern aufbewahrt, zusammen mit den Akten der Arztegesellschaft des Kantons Bern aus den ersten 100 Jahre ihres Bestehens, 1809-1909.

- Wehrli GA. Die Wundärzt und Bader Zürichs als zünftige Organisation. Zürich; 1931. 